\title{
Possible Causes of Asymmetry in the Mössbauer Spectra of Brain Tissue
}

\author{
K. Szlachta* And J. GatazKa-Friedman
}

Faculty of Physics, Warsaw University of Technology, Koszykowa 75, 00-662 Warszawa, Poland

Iron present in the human brain plays important role in neurodegenerative diseases like Parkinson and Alzheimer diseases. Brain iron is mainly stored within globular protein called ferritin. Mössbauer spectra of brain tissue are asymmetric doublets at $300 \mathrm{~K}$ and $90 \mathrm{~K}$. This asymmetry is slightly bigger in the case of samples taken from diseased subjects. Reason for this is still unknown. Few possible causes of aforesaid difference had been discussed. Nanometer-size of ferritin iron cores, pathological protein influence were taken into account. It was also discussed if experimentally available statistics is sufficient to obtain reliable asymmetry coefficient from Mössbauer spectra. Physical experiment were reproduced by means of computer simulations. Nevertheless, the reason for the asymmetry has not been completely elucidated yet.

PACS: $87.64 . \mathrm{kx}$

\section{Introduction}

Iron present in the human body, except in blood, is mainly stored within the ferritin. Ferritin is a globular protein composed of two types of chains, so called $\mathrm{H}$ and L chains. Sizes of ferritin's iron cores are different in different organs: in the brain its inner diameter is $c a .3 .5 \mathrm{~nm}$ and in the liver is $c a .6 \mathrm{~nm}$.

Mössbauer spectra of brain tissue, both, at $90 \mathrm{~K}$ (fresh samples) and at RT (lyophilized samples) are asymmetric doublets. An additional parameter, the asymmetry coefficient, had been introduced [1] to assess the magnitude of the spectrum asymmetry. Spectra had been fitted with two singlets with the same area but different width. Ratio of these widths was defined as the asymmetry coefficient. This asymmetry coefficient is significantly bigger in case of neurodegenerated brain structures. Physical reasons for this phenomenon have not yet been elucidated.

Low iron content in the human brain tissue, about $200 \mu \mathrm{g} / \mathrm{g}$ and count number experimental limit generates high noise to signal ratio. Additionally number of obtainable samples is limited. Furthermore, due to interindividual differences, samples taken from control or diseased subjects slightly differ. Parameters of this distribution are unknown. It is very important to check whether experiment typical for biological samples could give reliable results.

Experimentally measured coefficients of asymmetry $c_{A}$ for substantia nigra (SN) [2] are $1.06 \pm 0.02$ for 21 control samples and $1.14 \pm 0.03$ for 9 Parkinson's disease samples.

\footnotetext{
* corresponding author; e-mail: karolszl@if.pw.edu.pl
}

For globus pallidus [3] values of $c_{A}$ are $1.08 \pm 0.02$ for 12 control samples and $1.12 \pm 0.03$ for 10 Progressive Supranuclear Palsy disease samples, which is a type of parkinsonism.

\section{Asymmetry by statistical chance}

\subsection{Mathematical model of the Mössbauer spectra of brain tissue}

The Mössbauer spectrum $S$ of brain tissue was defined to be a sum of three component: Mössbauer signal, background level, and statistical noise. The Mössbauer signal is an asymmetric doublet being the sum of two Lorenzian lines $L$ with the same area $A$ but different half width at half maximum (HWHM) $w$ determined by asymmetry coefficient $c_{A}$. The positions of these lines $\left(v_{-}\right.$and $\left.v_{+}\right)$ are determined by quadrupole spliting (QS) and isomer shift (IS) of a simulated Mössbauer spectrum. The background level $B G$ is a constant value. Statistical noise $N$ is a random number taken from normal distribution [4] with mean value zero and variance equal to square root of background level.

$$
\begin{aligned}
& S(v)=B G+N\left(0, B G^{1 / 2}\right)-L\left(v-v_{-}, w, A\right) \\
& -L\left(v-v_{+}, w c_{A}, A\right)
\end{aligned}
$$

To generate a spectrum one has to determine the following set of input parameters: $B G, w, c_{A}, A, I S, Q S$, maximum value of source velocity $\left(v_{\max }\right)$ and number of channels $\left(n_{c}\right)$.

For all simulations, if not stated otherwise, these parameters values were: $B G=36 \times 10^{6}, w=0.23 \mathrm{~mm} / \mathrm{s}$, $A=41.5 \times 10^{3}$ counts $\times$ channel, $I S=0.47 \mathrm{~mm} / \mathrm{s}$, $Q S=0.67 \mathrm{~mm} / \mathrm{s}, v_{\max }=3.5 \mathrm{~mm} / \mathrm{s}$ and $n_{c}=256$. In order to precisely reproduce experimental conditions 
the number of simulated spectra was twenty. These values are typical for brain tissue Mössbauer spectra parameters and experimental conditions. Three calculated Mössbauer spectra of SN with different asymmetry are shown in Fig. 1. All spectra were fitted by means of Recoil program [5], in the same way as brain tissues spectra usually are. Experimental errors of standard deviation were estimated by means of confidence interval method (assumed confidence level corresponding to single standard deviation $\alpha=100 \%-68.3 \%$ ).

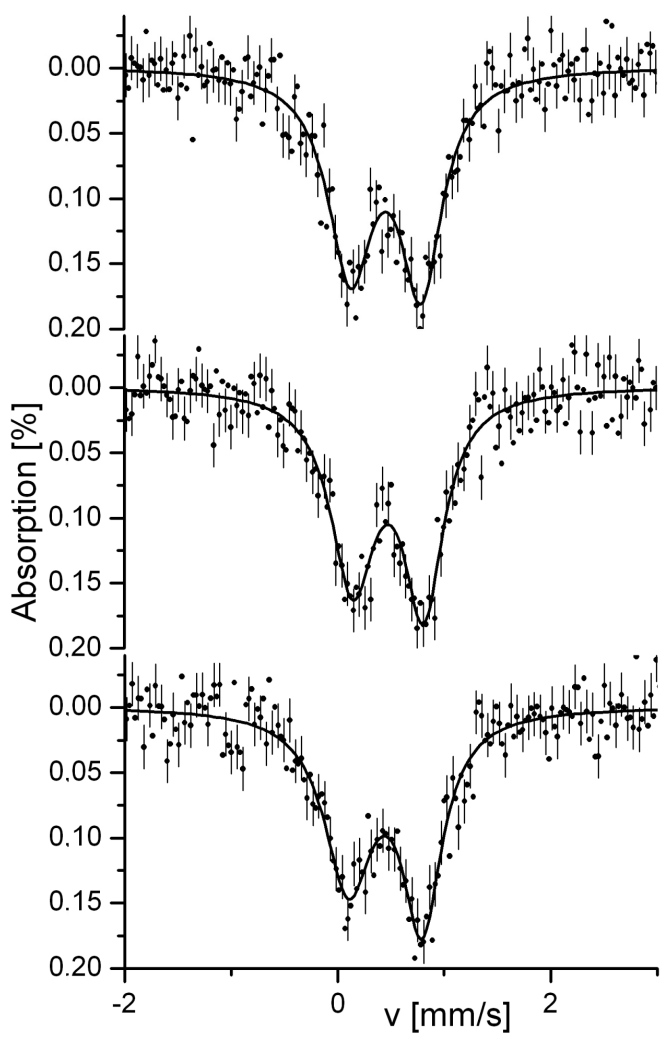

Fig. 1. Three simulated SN Mössbauer spectra with different asymmetry: (top) $c_{A}=1.07$, (middle) $c_{A}=$ 1.12 and (bottom) $c_{A}=1.21$.

Performed tests, not enclosed in this paper, proved that programs based on this model are working properly.

\subsection{General noise influence on Mössbauer parameters}

To assess noise influence on Mössbauer parameters twenty Mössbauer spectra with the same input parameters were calculated and then analyzed. Obtained Mössbauer parameters were normally distributed - see Fig. 2 . Mean values of obtained parameters were, within limits of error, equal to input parameters. Standard deviations of obtained parameters distributions are fully noise generated, see Table I.

\subsection{Does $c_{A}$ distribution depend on asymmetry?}

To investigate the properties of $c_{A}$ distribution two factors have been considered, the first of which was spectrum asymmetry. Four simulations (twenty Mössbauer
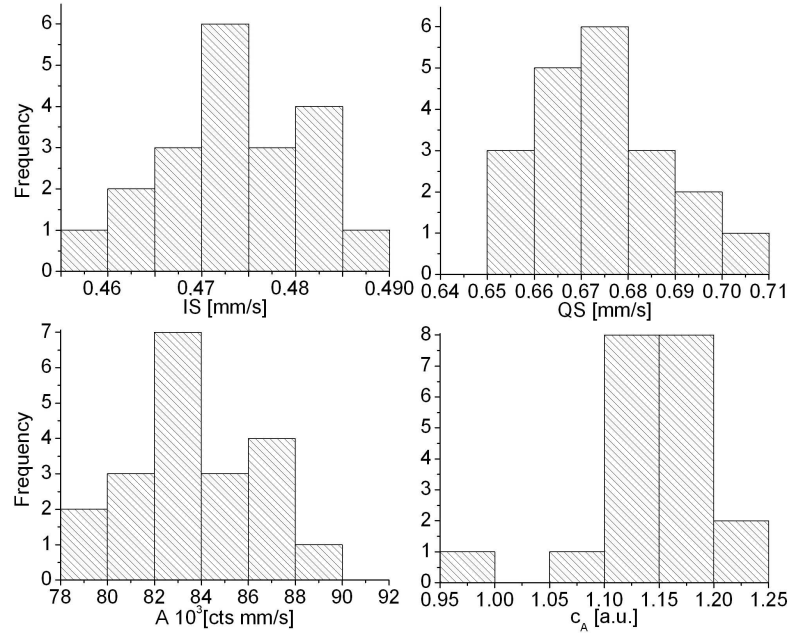

Fig. 2. Noise generated distributions of Mössbauer parameters obtained from simulated spectra: $I S$ (top left), $Q S$ (top right) $A$ (bottom left) and $c_{A}$ (bottom right).

TABLE I

Parameters of distributions obtained from simulated spectra and input parameters values.

\begin{tabular}{l|c|c|c|c|c|c|c}
\hline \hline & $\begin{array}{c}I S \\
{[\mathrm{~mm} / \mathrm{s}]}\end{array}$ & $\begin{array}{c}Q S \\
{[\mathrm{~mm} / \mathrm{s}]}\end{array}$ & $\begin{array}{c}B G \\
{[\mathrm{counts}]}\end{array}$ & $\chi^{2}$ & $\begin{array}{c}w \\
{[\mathrm{~mm} / \mathrm{s}]}\end{array}$ & $c_{A}$ & $A$ \\
\hline Input & 0.470 & 0.670 & 36000000 & - & 0.23 & 1.13 & 83240 \\
\hline Mean & 0.473 & 0.673 & 36000200 & 0.98 & 0.234 & 1.14 & 83700 \\
\hline s.d. & 0.008 & 0.01 & 500 & 0.08 & 0.01 & 0.05 & 3000
\end{tabular}

spectra each) were calculated and then analysed. Each simulations input parameters set differs in $c_{A}$. Coefficients and obtained results are presented in Table II. Calculated experimental errors of standard deviations is 0.004. Within limits of experimental error obtained $\sigma_{c}$ are the same for all simulations and its mean value equals to $0.060 \pm 0.003$. Obtained from distribution mean value of $c_{A}$ is equal to input parameter within limit of experimental error for all simulations.

TABLE II

Parameters of distributions obtained for four simulations and input asymmetry coefficient $c_{A}$.

\begin{tabular}{l|c|c|c|c}
\hline \hline Input & 1.00 & 1.07 & 1.13 & 1.20 \\
\hline Mean & 1.013 & 1.080 & 1.140 & 1.191 \\
\hline s.d. & 0.054 & 0.062 & 0.063 & 0.060
\end{tabular}

\subsection{Does asymmetry depends on the signal to noise ratio?}

Second of mentioned factors is the signal to noise ratio $(\mathrm{S} / \mathrm{N})$. Simulations were performed as previously but one hundred Mössbauer spectra were calculated. Simulations input parameters differ in $B G$. The asymmetry coefficient was taken to be 1.13 . Obtained $c_{A}$ dis- 
tributions parameters are presented in Table III. As expected, standard deviation of $c_{A}$ is inversely proportional to the square root of background level.

TABLE III

Parameters of $c_{A}$ distributions vs. background level.

\begin{tabular}{l|c|c|c|c|c|c|c}
\hline \hline$B G\left[\times 10^{6}\right]$ & 5 & 10 & 20 & 50 & 80 & 100 & 200 \\
\hline Mean $c_{A}$ & 1.26 & 1.19 & 1.16 & 1.22 & 1.21 & 1.21 & 1.19 \\
\hline s.d. & 0.12 & 0.12 & 0.08 & 0.040 & 0.033 & 0.027 & 0.022
\end{tabular}

\subsection{Could asymmetry be fully noise generated?}

Noise generated standard deviation of $c_{A}(0.060)$ is less than obtained from analysis of measurements (0.09) [2]. Let us assume that observed distribution of $c_{A}$ is a convolution of two independent Gaussian distributions: noise generated distribution with mean value 0 and $\sigma_{c}$ obtained in second simulation, and interindividual differences distribution with unknown parameters. Thus, by deconvolution of these distributions, parameters of the natural distribution could be estimated. The natural distribution mean value is equal to measured value because the noise distribution mean value equals zero. Natural variance is the difference between measured variance and noise generated variance. This result indicates that asymmetry coefficients for diseased and control samples are different, even with a higher probability than it was originally [2] calculated.

\section{Possible physical reasons of the asymmetry}

The motivation behind introducing an asymmetry coefficient had been to assess the spectrum asymmetry and to compare magnitude of it in Mössbauer spectra of samples taken from healthy and diseased subjects. However, no physical meaning has been attributed to this parameter so far.

\subsection{Effect of ferritin core's size}

Ferritin iron cores present in the human brain tissue are very small particles $\sim 3 \mathrm{~nm}$ [5]. They are twice as small in diameter as iron cores in ferritin present in the human liver or spleen. Mineralogical structure of ferritin is reported to be mainly ferryhydrite-like [6-7]. Ferryhydrite is a very ubiquitous iron oxide, well known as a rust. It is usually composed of $3-8 \mathrm{~nm}$ monocrystals often together with other iron oxides like hematite and goethite.

Assuming a ferryhydrite-like structure and spherical shape of iron ferritin core, the percentage of surface atom can be estimated. Ferryhydrite cell parameters [8] are: $a=0.5 \mathrm{~nm}$ and $c=0.94 \mathrm{~nm}$. There are four iron atoms positions within a cell but there are only two atoms on average. So the mean volume occupied by single iron atom is $11.75 \times 10^{-2} \mathrm{~nm}^{3}$. Knowing the core diameter one can estimate number of iron atoms present within the core and the percentage of atoms that are located in the outer part of the core.

These simple estimation showed that single molecule of ferritin in the brain contains fifteen times less iron atoms that single molecule of ferritin in the liver (140 vs. 2100). This result is consistent with blocking temperatures obtained for different ferritins [9].

It turned out that surface of ferritin iron core contain about $60 \%$ iron atoms in the case of brain ferritin and $25 \%$ in the case of liver ferritin. For example a forty nanometre particle of ferrihydrite consists of almost three hundred thousand iron atoms and only $c a .1 \%$ of which could be attributed to the surface. This estimation suggest that, in case of very small particles, the inner core with a structure similar to those of the bulk material covered with a surface mantle shielding outer influences is not a good model of such an object.

\subsection{Protein influence}

As mentioned ferritin is composed of $\mathrm{H}$ and $\mathrm{L}$ chains. $\mathrm{H} / \mathrm{L}$ ratio obtained for different organs defines not only protein chemical properties but also its core size. Interesting parameters [10] of different ferritins are put together in Table IV.

TABLE IV

Comparison of natural ferrihydrite and ferritins features. Ferritins comes from liver and three part of the human brain: hippocampus (HIP), substantia nigra (SN), and globus palliduss (GP). Parameter "size" is diameter of ferritin core or diameter of ferrihydrite nanaparticles respectively. Experimental errors of $I S$ and $Q S$ are $0.02 \mathrm{~mm} / \mathrm{s}$.

\begin{tabular}{l|c|c|c|c|c}
\hline \hline \multicolumn{2}{l|}{ Tissue } & $\mathrm{H} / \mathrm{L}$ & $\begin{array}{c}\text { Size } \\
{[\mathrm{nm}]}\end{array}$ & $\begin{array}{c}I S \\
{[\mathrm{~mm} / \mathrm{s}]}\end{array}$ & $\begin{array}{c}Q S \\
{[\mathrm{~mm} / \mathrm{s}]}\end{array}$ \\
\hline \multirow{3}{*}{ Brain } & HIP & $14.5 \pm 8$ & $3.1 \pm 0.5$ & 0.47 & 0.69 \\
\cline { 2 - 6 } & SN & $5.5 \pm 0.5$ & $3.7 \pm 0.5$ & 0.47 & 0.67 \\
\cline { 2 - 6 } & GP & $5.0 \pm 0.4$ & $3.5 \pm 0.2$ & 0.46 & 0.70 \\
\hline Liver & $0.40 \pm 0.04$ & $6.0 \pm 0.5$ & 0.45 & 0.60 \\
\hline $\begin{array}{l}\text { Natural } \\
\text { ferrihydrite [10] }\end{array}$ & - & $3-8$ & 0.35 & 0.75
\end{tabular}

There are no significant differences in Mössbauer parameters or ferritin iron cores diameters within the brain, despite the fact that the HIP $\mathrm{H} / \mathrm{L}$ ratio is almost three times higher than in SN or GP. Brain tissue compared to the liver tissue, shows lower $\mathrm{H} / \mathrm{L}$ ratio and bigger ferritin cores. There is also a difference in Mössbauer parameters. Both brain and liver tissues have different Mössbauer parameters from natural ferryhydrite.

\subsection{Ferritin dysfunction}

Results obtained by microstructure analysis methods suggest that there is significant difference in crystallographic structure between healthy and diseased ferritin iron cores $[7,11]$. Some minerals present in diseased ferritin contain $\mathrm{Fe}^{2+}$ atoms. This finding may suggest dysfunction of ferritin $\mathrm{H}$ chains and presence of divalent iron within the core. 


\section{Conclusions}

Results obtained by numerical simulations of Mössbauer spectra proved that a high signal to noise ratio is not able to cause the observed asymmetry. Nevertheless its influence on the obtained results is significant and always has to be carefully considered.

Different $\mathrm{H} / \mathrm{L}$ ratios observed in different parts of the body correlates with different structure of the ferritin. It seems that the lower this ratio is, the bigger are ferritins' cores. However, a relatively big difference in brain ferritins structure does not reflects significant difference in core diameters or Mössbauer parameters. It might be because of relatively big experimental errors due to low $\mathrm{S} / \mathrm{N}$ ratio. Brain and liver tissue comparison revealed following correlations: the lower $\mathrm{H} / \mathrm{L}$ ratio is the smaller core and lower $Q S$ are. For brain and liver ferritin $I S$ has the same value. Mössbauer parameters of ferritin cores are different than those of natural ferrihydrite.

Iron cores of brain ferritin are very small. Excluding the surface, which should be enough to shield influence of the surroundings, the single iron core consists of about 70 iron atoms that are able to build structure similar to crystal structure of bulk material. Additionally, it had been found that [6] "as particle size decreases, individual characteristics of the different iron oxides gradually disappear until finally only fundamental structural elements of short range order common to all (iron oxides) remain". Thus, asymmetry could be explained by a slight difference in this order.

It was suggested [7] that diseased ferritin cores have different crystalline structure because they may consist of divalent iron. Mössbauer investigations clearly show that if divalent iron is present in ferritin it cannot be more than $5 \%[12]$ of total iron. Moreover superposition of typical divalent and trivalent iron doublets cannot create asymmetric doublets as observed for brain tissue.

It seems that although statistical fluctuation affects measurements and obtained results, spectrum asymme- try cannot be explained by that. Observed difference in core structure, understood as difference in local order, together with protein shell influence may probably be the cause of observed asymmetry. Presence of divalent iron within the core is very doubtful. More experimental data is needed to prove or reject this hypothesis. It is however still unclear what is the primary cause of the disease.

\section{References}

[1] J. Gałązka-Friedman, E.R. Bauminger, A. Friedman, Hyperfine Interact. 141/142, 267 (2002).

[2] J. Gałązka-Friedman, E.R. Bauminger, D. Koziorowski, A. Friedman, Biochim. Biophys. Acta 1688, 130 (2004).

[3] J. Gałązka-Friedman, E.R. Bauminger, K. Szlachta, K. Schweitzer, Z. Wszolek, D. Dickson, A. Friedman, Acta Phys. Pol. A 115, 545 (2009).

[4] W.H. Press, S.A. Teukolsky, W.T. Vetterling, B.P. Flannery, Numerical Recipes in $C$ The Art of Scientific Computing, Second Edition, Cambridge University Press, Cambridge 2002.

[5] http://www.isapps.ca/recoil/.

[6] E. Murad, U. Schwarzmann, Am. Mineral. 65, 1044 (1980).

[7] C. Quintana, L. Gutierrez, Biochim. Biophys. Acta 1800, 770 (2010).

[8] J.L. Lambor, J. Puziewicz, Am. Mineral. 75, 431(1990).

[9] G.C. Papaefthymiou, Biochim. Biophys. Acta 1800 886 (2010).

[10] J. Gałązka-Friedman, Hyperfine Interact. 189, 31 (2009).

[11] J.M. Cowley, D.E. Janney, R.C. Gerkin, P.R. Buseck, J. Struct. Biol. 131, 210 (2000).

[12] J. Gałązka-Friedman, E.R. Bauminger, A. Friedman, M. Barcikowska, D. Hechel, I. Nowik, Mov. Disord. 11, 8 (1996). 